\title{
Nonlinear transport in semi-insulating GaAs: Critical fields and nucleation of high-field domains
}

\author{
B. Willing and J. C. Maan* \\ Grenoble High Magnetic Field Laboratory, Max-Planck-Institut für Festkörperforschung, 25 avenue des Martyrs, F-38042 \\ Grenoble, France
}

(Received 10 November 1993)

\begin{abstract}
Time-resolved local voltage profiling in semi-insulating $\mathrm{GaAs}$ by electro-optics shows spontaneous current oscillations being caused by both contact domains and propagating domains in the bulk. In particular, we prove that the critical electric fields in the bulk are much higher than the average field inferred from geometry and exceed the threshold field for the Gunn effect. The dynamics of domain propagation are governed by the interaction between two different oscillatory systems, namely the contact region and bulk GaAs.
\end{abstract}

The current versus electric-field $(j-E)$ characteristic of semi-insulating (SI) GaAs shows, at high bias voltages, an $N$-shaped region which demonstrates negative differential conductivity (NNDC). The uniform electric field distribution of a sample biased into the NNDC region is unstable and therefore splits into domains of low and high electric field. The high-field domains form spontaneously at the cathode, drift through the sample, and are eventually annihilated at the anode. These processes are connected to spontaneous low-frequency oscillations (LFO, $0.1-100 \mathrm{~Hz}$ ) of the total current under the application of a constant voltage $\mathrm{V}_{\text {appl }}$. LFO in SI GaAs and domain formation have been known since the early sixties, ${ }^{1}$ but the recent interest stems mainly from the fact that period doubling and bifurcation, eventually leading to chaotic behavior $^{2-5}$ is observed in this system. These LFO domains are quite different from the well understood Gunn domains, since their domain drift velocity is about six orders of magnitude lower. Furthermore, the threshold field for Gunn domains is $E=3.1 \times 10^{5} \mathrm{~V} \mathrm{~m}^{-1}$, while critical fields for LFO have been estimated to be below $E=1 \times 10^{5} \mathrm{Vm}^{-1}$. To explain the LFO domains, an alternative model based on field-enhanced trapping has been put forward. ${ }^{5-7}$ However, as most experiments so far either have been terminal measurements ${ }^{2-4,8}$ or have lacked significant temporal resolution, ${ }^{9-12}$ experimental data has never been accurate enough to test theory.

In this paper, we present measurements of the local voltages during the formation and the propagation of the domains as a function of time by using an electrooptic imaging technique. This provides an insight into the mechanisms of domain formation and LFO. In particular, the investigation of static voltage profiles just below the onset of oscillation reveals the existence of different critical field values for cathode region and bulk GaAs. Both of them are substantially higher than the configurational electric field ( $E_{\text {conf }}$ is the applied voltage per sample length), which up to now has been the only measure for critical fields. This puts the existing theories for bulk domain formation-based on $E_{\text {conf -into question. }}$ Furthermore, time-resolved voltage profiles during a LFO cycle show how the overall electric behavior is driven by the coupling of the nonlinear $j-E$ characteristics of bulk and contacts.

Our experimental setup is based on the longitudinal electro-optic effect by which we can read the voltage distribution of a surface in terms of a phase shift distribution. ${ }^{13}$ The latter is introduced to a linearly polarized, collimated laser beam by an electro-optic BSO crystal with a dielectic mirror on one and a transparent electrode on the other side, placed on top of the sample (supplied by K. de Kort, Philips NatLab, Eindhoven, The Netherlands). The phase shift is derived from the intensity distribution observed with an externally triggered CCD camera and a digital image processing system; voltages are calibrated putting the sample on a uniform potential with respect to the transparent electrode. The voltage resolution is about $5 \%$ of the maximum detected voltage, spatial resolution is $1 \times 10^{-5} \mathrm{~m}$, and temporal resolution several microseconds. Samples with a cross section of $5 \times 0.5 \mathrm{~mm}^{2}$ and lengths of $2-6.5 \mathrm{~mm}$ were cut from Sumitomo wafers of undoped, SI GaAs. Parallel contacts were evaporated using different materials $(\mathrm{Au} / \mathrm{Ge} / \mathrm{Ni}, \mathrm{Au}$ and $\mathrm{Sn})$; all except the Sn contacts were alloyed. The samples were biased with a low noise power supply in series with a load resistor of $R_{\text {load }}=200 \mathrm{k} \Omega$. The latter was used as a current monitor, having about $0.1 \%$ of the sample's resistance and thus providing a sufficiently steep load line. Experiments were performed at stabilized room temperature without illumination of the sample (the dielectric mirror preventing the probing light from reaching the sample).

All samples showed similar current versus voltage (I$V_{\text {appl }}$ ) characteristics revealing three distinct regimes [Fig. 1(a)] as reported by other authors. ${ }^{2,3,8,9,11}$

(i) At low $V_{\text {appl }}$, the characteristic is slightly sublinear, but smooth. An optical voltage profile taken in this regime shows a constant voltage drop over the sample, with a distinctly enhanced field near the cathode.

(ii) Above a certain applied voltage $V_{c, O}$, small sinoidal oscillations in the range of $1-100 \mathrm{~Hz}$ appear in the current, the amplitude of which grows smoothly with $V_{\text {appl }}$. The images show weak, fluctuating domains forming at some points of the cathode border. They fade out within 
a distance of $0.5 \mathrm{~mm}$ from the cathode and can be detected visually even before the current oscillations exceed the noise level ( $40 \mathrm{~dB}$ below the signal level). Therefore, we define $V_{c, O}$ as the voltage just below observation of contact domains on the screen.

(iii) At a critical voltage $V_{c, D}$, the sinoidal current oscillations sharply change into pulselike oscillations between two levels of total current (Fig. 2). The voltage profile shows distinct domains forming over the whole width of the cathode and propagating towards the anode, where they collapse and the cycle starts again. During propagation, the domain changes its shape slightly at certain locations in the sample. This fits well with Ref. 10 which reports an interaction between the domain and dislocation structures in the sample.

The described profiles imply that the sample can be seperated into three regions: a cathode region under re-
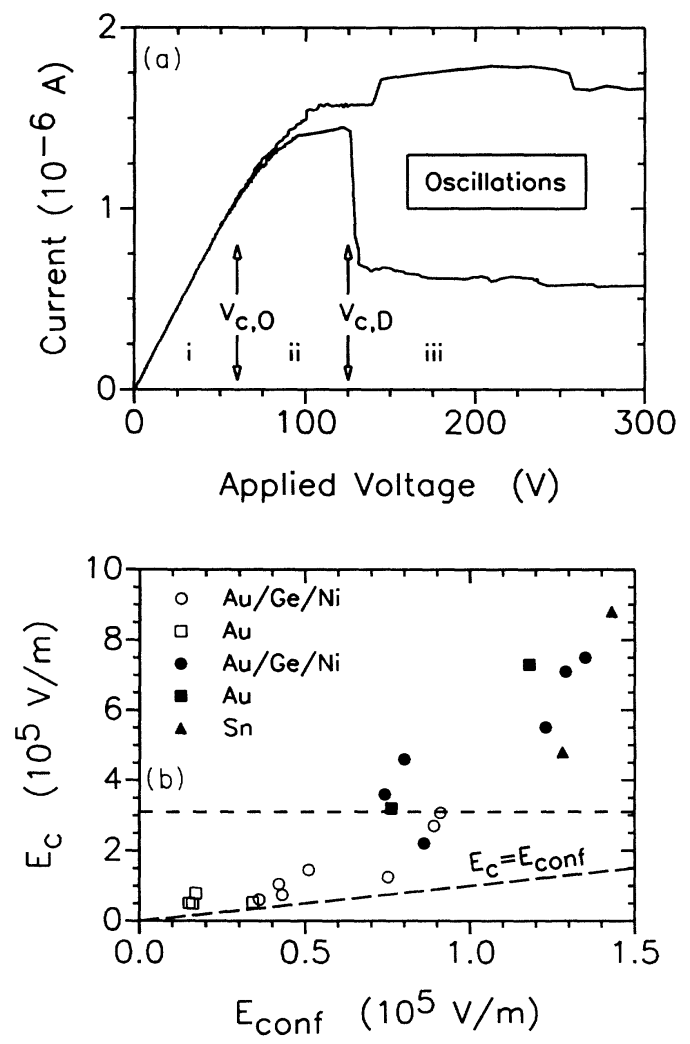

FIG. 1. (a) A typical current versus voltage characteristic of SI GaAs shows three regimes: (i) (sub-)linearity $\left(V_{\text {appl }} \leq V_{c, O}\right)$, (ii) smoothly growing, small oscillations $\left(V_{c, O} \leq V_{\text {appl }} \leq V_{c, D}\right)$, and (iii) sharp onset of large oscillations $\left(V_{\text {appl }} \geq V_{c, D}\right)$. Maximum and minimum current values during oscillation are indicated by the two characteristics. (b) Comparison between critical fields $E_{c}$ deduced at $V_{c}$ for various contacts of different materials (the two contacts of a sample mutually acting as a cathode) and the assumption of the according uniform configurational field $\left(E_{c}=E_{\text {conf }}=V_{c} / l, l\right.$ : contact spacing). Open dots indicate the onset of contact domains at $E_{c, O}$, full dots the nucleation of bulk domains at $E_{c, D}$. The dotted horizontal line refers to the critical field for Gunn effect. Note the separate ranges of $E_{c, O}$ and $E_{c, D}$, and that all contacts show $E_{\text {conf }}<1.5 \times 10^{5} \mathrm{~V} \mathrm{~m}^{-1}$. versed bias, a bulk region, and an anode region under forward bias. Any repartition of $V(x)$ over these regions has to comply with the boundary condition of a fixed $V_{\text {appl }}$.

To determine the critical fields $E_{c, O}$ and $E_{c, D}$ connected to $V_{c, O}$ and $V_{c, D}$, we deduced $E(x)$ from the static voltage profiles computing $d V / d x$. $E_{c, O}$ and $E_{c, D}$ were then taken as the maximum values of $\left.E(x)\right|_{V_{c, O}}$ and $\left.E(x)\right|_{V_{c, D}}$, respectively. The results for 10 contacts acting as a cathode are shown in Fig. 1(b) and compared to $E_{\text {conf }}$ (normalization of contact spacing). It can clearly be seen that $E_{\text {conf }}$ underestimates $E_{c, O}$ significantly because a large portion of the voltage drops over the cathode region. For $E_{c, D}$, the usual approximation by $E_{\text {conf }}$ does not hold at all. As can be seen from Fig. 1(b), $E_{c, O}$ and $E_{c, D}$ obviously form two separates ranges of field values. Contact oscillations basically set in at $E_{c, O}=0.5-1.25 \times 10^{5} \mathrm{~V} \mathrm{~m}^{-1}$ and the corresponding weak domains fade out as they enter the bulk, where their electric field is still too low to nucleate stable domains. Since they change the current density only locally near the cathode, the total current shows small sinoidal or noisy oscillations. Bulk domains set in at fields $E_{c, D}=3.1$ $8.8 \times 10^{5} \mathrm{~V} \mathrm{~m}^{-1}$, where a propagating domain develops to a peak field much higher than the initial critical field. From the samples investigated, no connection between contact material and critical field can be drawn. However, it was found that only the not-alloyed Sn contacts, which are not supposed to have an extended contact region, do not show any contact oscillations below $E_{c, D}$.

The fact that the critical fields are much higher than approximated by the configurational field has important consequences on the interpretation of the results. The field values at which contact oscillations appear are indicative for nonlinear conductivity driven by generation and recombination of carriers through impact ionization and field-enhanced trapping involving midgap traps. This mechanism was developed to explain bulk domains, ${ }^{5}$ but our data show that it is rather applicable to the contact regions. The measured critical field values for the bulk domains are sufficiently high to accelerate free carriers from the $\Gamma$ to the $X$ minimum in the conduction band, which is the mechanism for the Gunn effect. On the other hand, the domain velocity is several orders of magnitude slower than Gunn domains which is typical for a mechanism involving traps. From the data it must, therefore, be concluded that both trapping and negative differential mobility play a role in LFO domains.

We now turn to the time evolution of a complete domain cycle at a fixed voltage in the pulselike regime. Figure 2 shows the electric field profiles $E(x)$ of a $6.45 \mathrm{~mm}$ long sample taken at discrete times of the current oscillations $I(t)$. It can clearly be seen how a domain propagates (with a velocity of $\approx 1.6 \times 10^{-2} \mathrm{~ms}^{-1}$ ) from the cathode to the anode while the current has a constant low value [Fig. 2(b)]. The decrease in velocity on the domain's approach to the anode has not been investigated more closely as it is of no direct importance for the present discussion. The domain is basically a high resistance region in the sample (high voltage for a low current) which limits the total current as long as it is in 
the bulk GaAs far from the contacts. The close-up on the current peak [Fig. 2(a)] shows that the fast rise of the current (within $\approx 10^{-3} \mathrm{~s}$ ) is connected to the arrival of the domain peak field $\left(E_{\text {peak }} \approx 12 \times 10^{5} \mathrm{~V} \mathrm{~m}^{-1}\right.$ for $\left.V_{\text {appl }}=1000 \mathrm{~V}\right)$ at the anode region. Although the anode should differ somewhat from the cathode in its $j$ - $E$ characteristic, the preceding section nevertheless implies that it has a similar low $E_{c, O}$ compared to the bulk. We, therefore, conclude that the anode has broken down under the very high $E_{\text {peak }}$ and can supply a high current, whereas the bulk remains under the stable condition of the low-field value outside a domain. The slow decay of the peak field at the anode (during $\approx 0.02 \mathrm{~s}$ ) is directly transferred to an increase of the electric field at the cath-

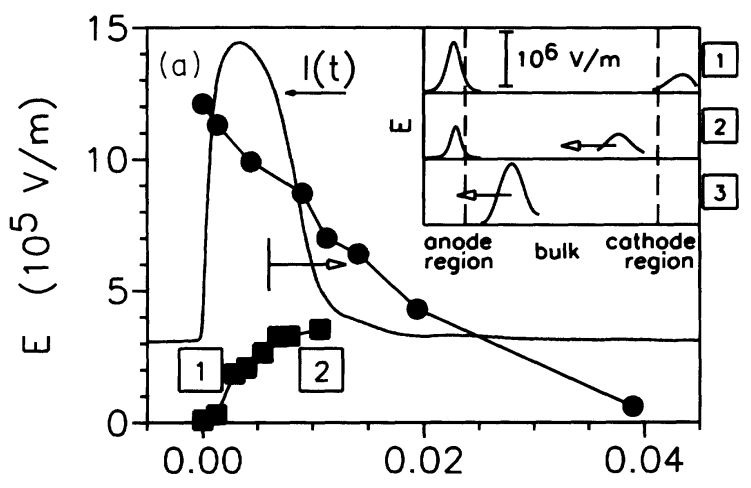

Time (s)

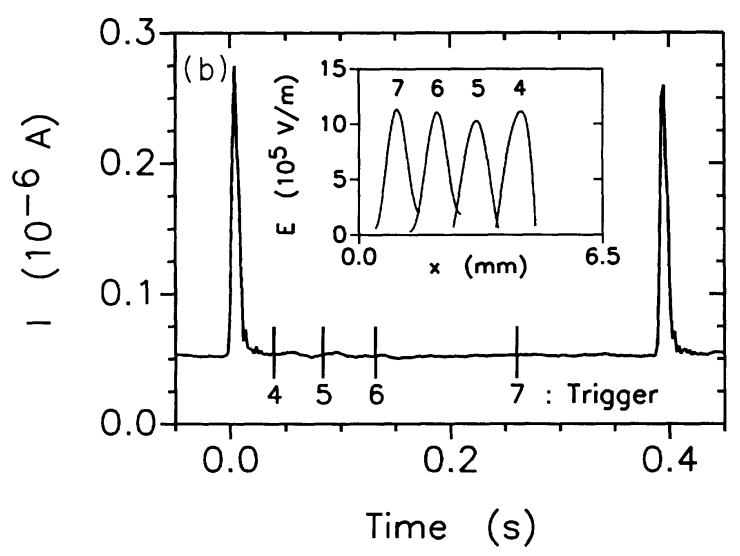

FIG. 2. (a) Temporal evolution of the maximum detected fields parallel to the current direction at the anode (circles, $x=0 \mathrm{~mm}$ ) and the cathode (squares, $x=6.45 \mathrm{~mm}$ ), $V_{\text {appl }}=1000 \mathrm{~V}$. At the anode, the arriving domain gradually disappears while its field is directly transferred to the cathode; an intermediate state of uniform electric field does not exist. The drop of the current peak (in arbitrary units) is linked to the penetration of the new domain into the bulk (indicated by the arrow) where it moves as a soliton towards the anode (b). The overall field distribution during domain nucleation, transit, and annihilation is given in the inset of (a). The corresponding $I(t)$ together with the according times $(1)-(7)$ is shown in (a) and (b). The time (3) corresponds to $t \approx 0.15 \mathrm{~s}$ and is offscale in (a). ode [inset in Fig. 2(a)]; an intermediate state of homogeneous $E_{\text {conf }}$ does not exist. As the cathode field exceeds $E_{c, D}=3.6 \times 10^{5} \mathrm{~V} \mathrm{~m}^{-1}$, a newly formed domain penetrates into the bulk which is directly accompanied by the drop of the current. The new domain still grows during the first part of its motion at the expense of the previous one, until the first one has completely disappeared. From this point on, the second domain proceeds as a soliton through the sample and the current has reached its constant low value. A similar observation of two domains in a sample has been made in ultrapure $p$-type Ge. ${ }^{14}$

Neglecting the influence of contacts during transit, the current is determined by $E_{\text {peak }}$ of the domain and the $j-E$ characteristic of bulk GaAs. On the other hand, during the fadeout of the old domain at the anode and the growth of the new one still fixed to the cathode, $E_{\text {peak }}$ and with it the current decay slowly along the $j$ - $E$ characteristic of the contact regions. Here we can neglect the contribution of the bulk. Principally, the domain peak field $E_{\text {peak }}$ switches the current between a low value given by $j\left(E_{\text {peak }}\right)$ of the bulk and a high value connected to $j\left(E_{\text {peak }}\right)$ of the anode region, but not between $j\left(E_{\text {peak }}\right)$ and $j_{\max }$ of the bulk $j-E$ characteristic.

The experimental results provide a complete insight into the behavior of the $I\left(V_{\text {appl }}\right)$ curve and the $I(t)$ dependence at different bias voltages. In its Ohmic part, all parts of the sample are stable. For voltages above $V_{c, O}$, $E_{c, O}$ is reached somewhere at the cathode which becomes unstable, whereas the bulk still remains stable. Finally, for $V_{\text {appl }}>V_{c, D}$, the critical field of bulk GaAs $E_{c, D}$ is exceeded. During an oscillation, the sample switches now between the two states "unstable contacts/stable bulk" and "stable contacts/unstable bulk."

The temporal evolution of a complete domain cycle can be described by two characteristic times; $t_{1}$ being the domain decay time at the anode and $t_{2}$ the transit time of the domain. $t_{1}$ mainly determines the duration of the current peak, since the current will drop when the first domain has decayed sufficiently to allow a next domain to enter the bulk, while $t_{2}$ measures the interval between current pulses. If $t_{1} \ll t_{2}$, the two states do not interfere and the period of oscillation is $t_{1}+t_{2}$. If $t_{1}$ approaches $t_{2}$, the new domain grows during a part of its motion (as described above), eventually still being below its full size before reaching the anode. It should be, therefore, possible ${ }^{3,5,9}$ to find a range of $t_{1}$ and $t_{2}$ which gives rise to nonlinear coupling between the two states, eventually leading to chaotic behavior.

In conclusion, we have presented time-resolved voltage profiles of domain formation in SI GaAs which reveal that the electrical behavior is governed by localized contact domains and propagating high-field domains. We show that critical fields for these latter domains are much higher than assumed previously and must exceed the threshold field for Gunn effect in order to incubate stable domains. This shows that present theories lack important features. The values for these stable domains are a bulk property and are not related to the contact material. The connection of $V(x, t)$ and $I(t)$ shows that the current oscillations are produced by the switching of the sample between the $j-E$ characteristics of the bulk and 
of the contacts. The interaction between these two oscillators provides a possible explanation for the observed chaotic behavior.
It is a pleasure to thank K. de Kort for his help with the experimental setup and J.Łusakowski for many helpful discussions.
${ }^{*}$ Present address: High Field Magnet Laboratory, Research Institut for Materials, B-Faculties, University of Nijmegen, 6500 Nijmegen, The Netherlands.

${ }^{1}$ B.K. Ridley and P.H. Wisbey, Br. J. Appl. Phys. 18, 761 (1967).

${ }^{2}$ G.N. Maracas, W. Porod, D.A. Johnson, and D.K. Ferry, Physica B 134, 276 (1985).

${ }^{3}$ J. Eusakowski, M. Ježewski, W. Knap, and W. Kuszko, Acta Phys. Pol. A 73, 183 (1988).

${ }^{4}$ J. Pǒzela, A. Namajunas, A. Tamaševičius, and J. Ulbikas, Appl. Phys. A 48, 181 (1989).

${ }^{5}$ E. Schöll, Phys. Scr. T29, 152 (1989).

${ }^{6}$ H.K. Sacks and A.G. Milnes, Int. J. Electron. 30, 49 (1971).

${ }^{7}$ M.F. Leach and B.K. Ridley, J. Phys. C 11, 2265 (1978).

${ }^{8}$ J. Łusakowski, Acta Phys. Pol. A 77, 391 (1990).
${ }^{9}$ G.N. Maracas, D.A. Johnson, R.A. Puechner, J.L. Edwards, S. Myhajlenko, H. Goronkin, and R. Tsui, SolidState Electron. 32, 1887 (1989).

${ }^{10}$ D.A. Johnson, S. Myhajlenko, J.L. Edwards, G.N. Maracas, R.J. Roedel, and H. Goronkin, Appl. Phys. Lett. 51, 1152 (1987).

${ }^{11}$ U. Rau, J. Peinke, J. Parisi, K. Kapierz, J. Lusakowski, and W. Knap, Phys. Lett. A 152, 356 (1991).

${ }^{12}$ H. Rajbenbach, J.M. Verdiell, and J.P. Huignard, Appl. Phys. Lett. 53, 541 (1988).

${ }^{13}$ A. Yariv, Quantum Electronics, 2nd ed. (Wiley, New York, 1975).

${ }^{14}$ A.M. Kahn, D.J. Mar, and R.M. Westervelt, Phys. Rev. B 43, 9740 (1991). 